\title{
Reply to Roe and Baker's comment on “Another look at climate sensitivity" by Zaliapin and Ghil (2010)
}

\author{
I. Zaliapin ${ }^{1}$ and M. Ghil ${ }^{2,3}$ \\ ${ }^{1}$ Department of Mathematics and Statistics, University of Nevada, Reno, USA \\ ${ }^{2}$ Geosciences Department and Laboratoire de Météorologie Dynamique (CNRS and IPSL), Ecole Normale Supérieure, \\ Paris, France \\ ${ }^{3}$ Department of Atmospheric \& Oceanic Sciences and Institute of Geophysics \& Planetary Physics, University of California, \\ Los Angeles, USA
}

Received: 2 September 2010 - Revised: 8 December 2010 - Accepted: 28 January 2011 - Published: 18 February 2011

\begin{abstract}
G. H. Roe and M. B. Baker (hereafter R\&B) claim that analysis of a global linear approximation to the climate system allows one to conclude that the quest for reliable climate predictions is futile. We insist that this quest is important and requires a proper understanding of the roles of both linear and nonlinear methods in climate dynamics.
\end{abstract}

We are grateful to R\&B for their Comment (Roe and Baker, 2011, RB11 hereafter) on Zaliapin and Ghil (2010, ZG10 hereafter) and to this journal for an opportunity to reply. First off, we are glad to acknowledge an important point of agreement with $\mathrm{R} \& \mathrm{~B}$, in that Roe and Baker (2007, RB07 hereafter) do indeed apply a global linear relationship to understand climate response, by allowing its slope to have a normal distribution. This removes the main concern of ZG10, namely that a linear study was presented as an analysis of a general climate model with nonlinear feedbacks.

RB11 correctly note that the "fact that the climate system is nonlinear does not preclude the use or value of linear analyses". The role of local linearization in studying the nonlinear equations of climate dynamics is well known (Ghil and Childress, 1987); what is less obvious is applicability of such a linearization over a wide range of parameter values.

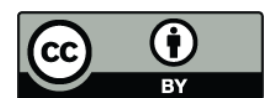

Correspondence to: I. Zaliapin (zal@unr.edu)
Let us return to the second-order Taylor expansion of the atmospheric radiation function $R=R(T, \alpha(T))$ :

$$
\Delta R=\frac{1-f}{\lambda_{0}} \Delta T+a(\Delta T)^{2}+\mathcal{O}\left((\Delta T)^{3}\right)
$$

where $1 / \lambda_{0}=\partial R /\left.\partial T\right|_{T=0}, \quad f=-\lambda_{0} F(0)$, and $F(T):=$ $(\partial R / \partial \alpha)(\partial \alpha / \partial T)$.

RB11 state that the higher-order terms in Eq. (1) are negligible and support this assertion by several estimates of the two quantities denoted by " $d f / d T$ " and " $-f^{\prime} /\left(2 \lambda_{0}\right)$ " in their Table 1. Ignoring some unfortunate inconsistencies e.g., $f$ is a constant and thus $d f / d T \equiv 0-$ columns 4 and 5 of their table refer in fact to $\lambda_{0} F^{\prime}(0)$ and $F^{\prime}(0) / 2$, where $F^{\prime} \equiv d F / d T$.

Several comments on Table 1 of RB11 are in order:

a. the higher-order terms represented by $\mathcal{O}\left((\Delta T)^{3}\right)$ are still being ignored;

b. an implicit assumption is made that $F^{\prime}(T) \equiv F^{\prime}(0)$, while $T$ is changing over $7-8 \mathrm{~K}-$ this assumption is incorrect but without it the linear model is invalid; and

c. $F^{\prime}(0) / 2$ is not a correct expression for $a$ in Eq. (1) - the correct formula is

$$
a=\left.\frac{1}{2} \frac{d^{2} R}{d T^{2}}\right|_{T=0}=\frac{1}{2}\left[\left.\frac{\partial^{2} R}{\partial T^{2}}\right|_{T=0}+F^{\prime}(0)\right] .
$$

We persist, therefore, in claiming that RB07's arguments do not apply to the study of climate sensitivity over a wide range of temperatures.

Published by Copernicus Publications on behalf of the European Geosciences Union and the American Geophysical Union. 
To emphasize, however, a more fundamental issue with R\&B's arguments, let us assume that the linear approximation $\Delta R=\Delta T(1-f) / \lambda_{0}$ is actually true. This approximation, or similar ones, have been studied since the 1970s (Wetherald and Manabe, 1975; Schlesinger, 1986). Some additional, substantial results are thus required to claim its validity for drawing conclusions about climate sensitivity in general.

At this point, RB07 state that "as is commonplace, we assume the errors in the feedback factors are normally distributed" (RB07, p. 631). Usually, the central limit theorem for the sum of random variables is invoked to assume a given variable is Gaussian. Why $f$ would be the limit of such a sum, rather than the result of nonlinear feedback mechanisms, remains to be explained.

Nevertheless, it is readily seen from Eq. (1) that using any broad and symmetric distribution of $f$ will result in a distribution of the response $\Delta T$ to a fixed change in $\Delta R$ that is strongly skewed towards large, positive $\Delta T$; this is RB07's main conclusion. A broad and symmetric distribution of $f$ is thus the key element of the RB07 arguments. Their statement that "the general features of our results do not depend on this assumption" (RB07, p. 631) is thus misleading: neither a narrow nor even a broad, but left-skewed distribution of $f$ will produce a right-skewed response in $\Delta T$.

To summarize, RB07's arguments are only as good as their two main assumptions:

a. linear climate response with constant, temperature- and time-independent slope; and

b. a broad and symmetric distribution of errors in the feedback factor $f$.

These assumptions are dubbed in RB07, p. 630, "far more fundamental" than the physics of clouds, the complex interaction among individual climate processes, and the chaotic, turbulent nature of the climate system. We leave it to the interested reader to decide whether s/he agrees.

Leaving these technicalities aside, our main concern is far from a particular model or assumed range of parameters. Namely, the far-reaching conclusions of RB07 about the impossibility of scientifically determining climate sensitivity have become separated from the supporting technical arguments, and have triggered substantial discomfort among climate researchers and policymakers alike. We can only refer here to the titles of several papers that cite RB07, e.g. Allen and Frame (2007); Mearns (2010); Schellnhuber (2008). It may indeed be appealing to give up the "quest" to solve the difficult problem of climate sensitivity, i.e. to find its causes and determine its range of values. But the history of modern science teaches us that tools more sophisticated than linear models are required in this quest.
To address some technical points of RB11:

i. Fitting a distribution is very different from finding physical reasons for it. Accordingly, the observation that the linear model in Fig. 1 of RB07 fits the distribution of climate sensitivity from climateprediction.net may be a neat statistical fact but its relationship to the physics of climate change, if any, has still to be explained.

ii. Wetherald and Manabe (1975) explicitly state (p. 2049) that their model may produce what is now called a "tipping point", similar to that in very simple energy balance models by M. I. Budyko, W. D. Sellers or I. M. Held and M. J. Suarez, among others.

iii. Section 3 of ZG10 illustrates a qualitative effect the presence of tipping points - that is unavoidable in climate models that take into account commonly accepted, nonlinear physics. One can easily tune the model parameters to obtain quantitatively realistic results, but this was not the point ZG10 wished to make; hence the discrepancy in the model's current Earth temperature.

iv. We prefer not to engage further in discussing matters of detail that are not related to our main message: the physics of the linear model considered in RB07 is conceptually wrong and technical details pertaining to its analysis do not improve the situation.

To conclude, we believe that - paraphrasing a famous quote, attributed to Mark Twain - rumors about the death of climate science have been greatly exaggerated. What researchers need to do is to stop panicking and continue, aggressively but calmly, the quest for predictive understanding of natural and anthropogenic climate change (Ghil, 2001; Held, 2005; McWilliams, 2007).

Edited by: D. Schertzer

Reviewed by: two anonymous referees

\section{References}

Allen, M. R. and Frame, D. J.: Call off the quest, Science, 318(5850), 582-583, doi:10.1126/science.1149988, 2007.

Ghil, M.: Hilbert problems for the geosciences in the 21 st century, Nonlin. Processes Geophys., 8, 211-211, doi:10.5194/npg-8211-2001, 2001.

Ghil, M. and Childress, S.: Topics in Geophysical Fluid Dynamics: Atmospheric Dynamics, Dynamo Theory and Climate Dynamics, Springer-Verlag, New York/Berlin/London/Paris/ Tokyo, 485 pp., 1987.

Held, I. M.: The gap between simulation and understanding in climate modeling, B. Am. Meteorol. Soc., 86, 1609-1614, 2005. 
McWilliams, J. C.: Irreducible imprecision in atmospheric and oceanic simulations, P. Natl. Acad. Sci. USA, 104, 8709-8713, 2007.

Mearns, L. O.: The drama of uncertainty, Climatic Change, 100(1), 77-85, doi:10.1007/s10584-010-9841-6, 2010.

Roe, G. H. and Baker, M. B.: Why is climate sensitivity so unpredictable?, Science, 318(5850), 629-632, doi:10.1126/science.1144735, 2007.

Roe, G. H. and Baker, M. B.: Comment on "Another Look at Climate Sensitivity" by I. Zaliapin and M. Ghil, Nonlin. Proc. Geophys., 18, 125-127, doi:10.5194/npg-18-125-2011, 2011.
Schlesinger, M. E.: Equilibrium and transient climatic warming induced by increased atmospheric $\mathrm{CO}_{2}$, Climate Dynam., 1, 3551, doi:10.1007/BF01277045, 1986.

Schellnhuber, H. J.: Global warming: Stop worrying, start panicking?, P. Natl. Acad. Sci. USA, 105(38), 14239-14240, 2008.

Wetherald, R. T. and Manabe, S.: The effect of changing the solar constant on the climate of a general circulation model, J. Atmos. Sci., 32, 2044-2059, 1975.

Zaliapin, I. and Ghil, M.: Another look at climate sensitivity, Nonlin. Processes Geophys., 17, 113-122, doi:10.5194/npg-17113-2010, 2010. 